\title{
SUBSEQUENT SINGULARITIES OF MEAN CONVEX MEAN CURVATURE FLOWS IN SMOOTH MANIFOLDS
}

\author{
QI DING
}

\begin{abstract}
For any $n$-dimensional smooth manifold $\Sigma$, we show that all the singularities of the mean curvature flow with any initial mean convex hypersurface in $\Sigma$ are cylindrical (of convex type) if the flow converges to a smooth hypersurface $M_{\infty}$ (maybe empty) at infinity. Previously this was shown (i) for $n \leq 7$, and (ii) for arbitrary $n$ up to the first singular time without the smooth condition on $M_{\infty}$.
\end{abstract}

\section{INTRODUCTION}

Singularities of mean curvature flow are unavoidable if the flow starts from a closed embedded hypersurface in Euclidean space. When the initial hypersurface is mean convex in Euclidean space, the mean curvature flow (level set flow) preserves mean convexity. So we sometimes call it mean convex mean curvature flow.

Huisken-Sinestrari obtained the convexity estimate for mean convex mean curvature flow [9-11] and the cylindrical estimate for mean curvature flow of two-convex hypersurface [11], respectively. In particular, any smooth rescaling of the singularity in the first singular time is convex by $[9,10]$. B. White in $[15,16]$ showed that any singularity of mean convex mean curvature flow which occurs in the first singular time, must be of convex type. Here, a singular point $x$ of the flow $M_{t}$ has convex type if

(1) any tangent flow at $x$ is cylindrical, namely, a multiplicity one shrinking round cylinder $\mathbb{R}^{k} \times \mathbb{S}^{n-k}$ for some $k<n$.

(2) for each sequence $x_{i} \in M_{t(i)}$ of regular points that converge to $x$,

$$
\liminf _{i \rightarrow \infty} \frac{\kappa_{1}\left(M_{t(i)}, x_{i}\right)}{H\left(M_{t(i)}, x_{i}\right)} \geq 0,
$$

where $\kappa_{1}, \kappa_{2} \cdots, \kappa_{n}$ are the principal curvatures with $\kappa_{1} \leq \kappa_{2} \cdots \leq \kappa_{n}$, and $H=\sum_{i} \kappa_{i}>$ 0 . Furthermore, White $[16,18]$ showed that all the singularities of mean convex mean curvature flow in Euclidean space are of convex type. And see $[1,8,14]$ for more results in this direction. On the other hand, Colding-Minicozzi [4] showed that the only singularities of generic mean curvature flow in $\mathbb{R}^{3}$ are spherical or cylindrical. In [3] Colding-IlmanenMinicozzi obtained a rigidity theorem for round cylinders in a very strong sense.

In the aspect of structure of the singular set of mean curvature flow, White [15] showed that parabolic Hausdorff dimension of the space-time singular set is $n-1$ at most for mean convex mean curvature flow in $\mathbb{R}^{n+1}$. When a mean curvature flow starts from a closed embedded hypersurface in $\mathbb{R}^{n+1}$ with only generic singularities, Colding-Minicozzi

The author is supported partially by 15ZR1402200. He would like to thank Haslhofer for discussion on non-compactness of translating solitons in the previous edition. He also would like to thank the referees for various suggestions to make this paper more readable. 
QI DING

[5] showed that their space-time singular set is contained in finitely many compact embedded $(n-1)$-dimensional Lipschitz submanifolds plus a set of dimension $n-2$ at most.

When the initial hypersurface is mean convex in an $n$-dimensional smooth manifold $\Sigma$, mean convexity is preserved by mean curvature flow $(\mathcal{M}, \mathcal{K})$ in $\Sigma$ in view of [15]. Let $\left(\mathcal{M}^{\prime}, \mathcal{K}^{\prime}\right)$ be any limit flow if $n \leq 7$ or a special limit flow if $n>7$, where $\mathcal{K}^{\prime}: t \in$ $\mathbb{R} \mapsto K_{t}^{\prime}$ (see [16] for the definition). Then $K_{t}^{\prime}$ is convex for every $t$ showed by White [16]. Furthermore, if $\left(\mathcal{M}^{\prime}, \mathcal{K}^{\prime}\right)$ is backwardly self-similar, then it is either (i) a static multiplicity 1 plane or (ii) a shrinking sphere or cylinder [16]. In this paper, we will show that $K_{t}^{\prime}$ is convex for every $t$ if $\left(\mathcal{M}^{\prime}, \mathcal{K}^{\prime}\right)$ is any limit flow for $n>7$ and the flow $\mathcal{M}$ converges to a smooth hypersurface (maybe empty) at infinity.

Theorem 1.1. Let $\mathcal{M}: t \in[0, \infty) \mapsto M_{t}$ be a mean curvature flow starting from a mean convex, smooth hypersurface in a complete smooth manifold. If $\lim _{t \rightarrow \infty}\left(\cup_{s>t} M_{s}\right)$ (maybe empty) is a smooth hypersurface, then all the subsequent singularities of $\mathcal{M}$ must have convex type.

Our proof heavily depends on Ilmanen's elliptic regularization and White's work on motion by mean curvature, where we give a delicate analysis for the second fundamental form of the corresponding translating soliton related to the considered mean curvature flow in a manifold. If either $\Sigma$ has nonnegative Ricci curvature or $\Sigma$ is simply connected with nonpositive sectional curvature, we can remove the smooth condition on the hypersurface $\lim _{t \rightarrow \infty}\left(\cup_{s>t} M_{s}\right)$, and get the same conclusion (see Corollary 3.3). This can be thought of as a generalization of Theorem 3 of White [18]. After this paper, Haslhofer-Hershkovits [7] got structure theorem of singularities of mean convex mean curvature flows in Riemannian manifolds by another method independently, where even they do not need the smooth condition of the flows at infinity.

\section{TRANSLATING SOLITONS FOR MEAN CURVATURE FLOW}

Let $(\Sigma, \sigma)$ be an $n$-dimensional smooth complete manifold with Riemannian metric $\sigma=\sum_{i, j=1}^{n} \sigma_{i j} d x_{i} d x_{j}$ in a local coordinate. Let $N$ denote the product space $\Sigma \times \mathbb{R}$ with the product metric

$$
\sigma+d t^{2}=\sum_{i, j} \sigma_{i j} d x_{i} d x_{j}+d t^{2}
$$

Let $\langle\cdot, \cdot\rangle$ and $\bar{\nabla}$ denote the inner product and the Levi-Civita connection of $N$ with respect to its metric, respectively. Set $\left(\sigma^{i j}\right)$ be the inverse matrix of $\left(\sigma_{i j}\right)$. Let $\partial_{x_{i}}$ and $E_{n+1}$ be the dual frame of $d x_{i}$ and $d t$, respectively. Denote $D f=\sum_{i, j} \sigma^{i j} f_{i} \partial_{x_{j}}$ and $|D f|^{2}=\sum_{i, j} \sigma^{i j} f_{i} f_{j}$ for any $C^{1}$-function $f$ on $\Sigma$. Let $\operatorname{div}_{\Sigma}$ be the divergence of $\Sigma$. Let $R$ and Ric denote the curvature tensor and Ricci curvature of $\Sigma$, respectively. Let $\bar{R}$ and $\overline{R i c}$ be the curvature tensor and the Ricci curvature of $N=\Sigma \times \mathbb{R}$, respectively.

Let $S$ be an $n$-dimensional smooth graph in $\Sigma \times \mathbb{R}$ with the graphic function $u$ and the induced metric $g$. In a local coordinate, $g=g_{i j} d x_{i} d x_{j}=\left(\sigma_{i j}+u_{i} u_{j}\right) d x_{i} d x_{j}$, and then $g^{i j}=\sigma^{i j}-\frac{u^{i} u^{j}}{1+|D u|^{2}}$, where $u^{i}=\sigma^{j k} u_{k}$. Let $\Delta, \nabla$ be the Laplacian and Levi-Civita connection of $(S, g)$, respectively. Let $\nu$ denote the unit normal vector field of $M$ in $N$ defined by

$$
\nu=\frac{1}{\sqrt{1+|D u|^{2}}}\left(-D u+E_{n+1}\right)
$$


Now we assume that $S$ is a translating soliton satisfying the following equation

$$
H+\lambda\left\langle E_{n+1}, \nu\right\rangle=0
$$

for some constant $\lambda>0$. The equation (2.2) is equivalent to

$$
\operatorname{div}_{\Sigma}\left(\frac{D u}{\sqrt{1+|D u|^{2}}}\right)+\frac{\lambda}{\sqrt{1+|D u|^{2}}}=0 .
$$

In a local coordinate, the equality $(2.3)$ can be rewritten as

$$
\sum_{i, j=1}^{n}\left(\sigma^{i j}-\frac{u^{i} u^{j}}{1+|D u|^{2}}\right) u_{i, j}+\lambda=0,
$$

where $u_{i, j}$ is the covariant derivative on $\Sigma$ with respect to $\partial_{x_{i}}, \partial_{x_{j}}$. Analog to Theorem 4.3 in [19], $S$ is an area-minimizing hypersurface with the weight $e^{-\lambda x_{n+1}}$ in $\Sigma \times \mathbb{R}$. By $\nabla u=D u-\langle D u, \nu\rangle \nu$ and (2.1), we get

$$
\left\langle E_{n+1}, \nabla u\right\rangle=-\langle D u, \nu\rangle\left\langle E_{n+1}, \nu\right\rangle=\frac{|D u|^{2}}{1+|D u|^{2}}=|\nabla u|^{2} .
$$

Choose a local orthonormal frame field $\left\{e_{i}\right\}_{i=1}^{n}$ in $S$, which is a normal basis at the considered point. Set the coefficients of the second fundamental form $h_{i j}=\left\langle\bar{\nabla}_{e_{i}} e_{j}, \nu\right\rangle$ and the squared norm of the second fundamental form $|A|^{2}=\sum_{i, j} h_{i j} h_{i j}$. Then the mean curvature $H=\sum_{i} h_{i i}$. Denote $\nabla_{i}=\nabla_{e_{i}}$ and $\bar{R}_{\nu j i k}=\left\langle\bar{R}_{\nu j} e_{i}, e_{k}\right\rangle=\left\langle-\bar{\nabla}_{\nu} \bar{\nabla}_{e_{j}} e_{i}+\right.$ $\left.\bar{\nabla}_{e_{j}} \bar{\nabla}_{\nu} e_{i}+\bar{\nabla}_{\left[\nu, e_{j}\right]} e_{i}, e_{k}\right\rangle$. From (2.2) and Codazzi equation $h_{j k, i}-h_{j i, k}=-\bar{R}_{\nu j k i}$, we have

$$
\begin{aligned}
\nabla_{i} \nabla_{j} H & =-\lambda \nabla_{i}\left\langle E_{n+1}, \nabla_{e_{j}} \nu\right\rangle=\lambda \nabla_{i}\left(\left\langle E_{n+1}, e_{k}\right\rangle h_{j k}\right) \\
& =\lambda\left\langle E_{n+1}, \nu\right\rangle h_{i k} h_{j k}+\lambda\left\langle E_{n+1}, e_{k}\right\rangle h_{j k, i} \\
& =\lambda\left\langle E_{n+1}, \nu\right\rangle h_{i k} h_{j k}+\lambda\left\langle E_{n+1}, e_{k}\right\rangle h_{j i, k}-\lambda\left\langle E_{n+1}, e_{k}\right\rangle \bar{R}_{\nu j k i} \\
& =\lambda\left\langle E_{n+1}, \nu\right\rangle h_{i k} h_{j k}+\lambda\left\langle E_{n+1}, \nabla h_{i j}\right\rangle-\lambda\left\langle E_{n+1}, e_{k}\right\rangle \bar{R}_{\nu j k i} .
\end{aligned}
$$

By Simons' identity (see [20] for instance), we have

$$
\begin{aligned}
\Delta h_{i j}= & \nabla_{i} \nabla_{j} H+H h_{i k} h_{j k}-|A|^{2} h_{i j}+H \bar{R}_{\nu i \nu j}-h_{i j} \overline{\operatorname{Ric}}(\nu, \nu) \\
& +\bar{R}_{k i k p} h_{j p}+\bar{R}_{k j k p} h_{i p}+\bar{R}_{k i j p} h_{k p}+\bar{R}_{p j i k} h_{k p}+\bar{\nabla}_{k} \bar{R}_{\nu j i k}+\bar{\nabla}_{i} \bar{R}_{\nu k j k} .
\end{aligned}
$$

From (2.2), substituting (2.6) to (2.7) we get

$$
\begin{aligned}
\Delta h_{i j}= & \lambda\left\langle E_{n+1}, \nabla h_{i j}\right\rangle-|A|^{2} h_{i j}-\lambda\left\langle E_{n+1}, e_{k}\right\rangle \bar{R}_{\nu j k i}+H \bar{R}_{\nu i \nu j}-h_{i j} \overline{R i c}(\nu, \nu) \\
& +\bar{R}_{k i k p} h_{j p}+\bar{R}_{k j k p} h_{i p}+\bar{R}_{k i j p} h_{k p}+\bar{R}_{p j i k} h_{k p}+\bar{\nabla}_{k} \bar{R}_{\nu j i k}+\bar{\nabla}_{i} \bar{R}_{\nu k j k} .
\end{aligned}
$$

Since $N$ is a product manifold with the product metric, then $\left\langle\bar{R}_{\nu j} e_{i}, E_{n+1}\right\rangle=0$ by Appendix A of [13]. Hence

$$
-\lambda\left\langle E_{n+1}, e_{k}\right\rangle \bar{R}_{\nu j k i}=\lambda\left\langle\bar{R}_{\nu j} e_{i}, E_{n+1}-\left\langle E_{n+1}, \nu\right\rangle \nu\right\rangle=-\lambda\left\langle E_{n+1}, \nu\right\rangle\left\langle\bar{R}_{\nu j} e_{i}, \nu\right\rangle=H \bar{R}_{\nu j i \nu} .
$$

Then we obtain

$$
\begin{aligned}
\Delta h_{i j}= & \lambda\left\langle E_{n+1}, \nabla h_{i j}\right\rangle-|A|^{2} h_{i j}-h_{i j} \overline{\operatorname{Ric}}(\nu, \nu) \\
& +\bar{R}_{k i k p} h_{j p}+\bar{R}_{k j k p} h_{i p}+2 \bar{R}_{k i j p} h_{k p}+\bar{\nabla}_{k} \bar{R}_{\nu j i k}+\bar{\nabla}_{i} \bar{R}_{\nu k j k} .
\end{aligned}
$$

and

$$
\Delta H=\lambda\left\langle E_{n+1}, \nabla H\right\rangle-\left(|A|^{2}+\overline{\operatorname{Ric}}(\nu, \nu)\right) H
$$


Let $\eta$ be a smooth function on $\mathbb{R}$ satisfying that $\eta(t)=t$ for $t \in(-\infty, 1], 1 \leq \eta \leq 2$ on $(1,3), \eta \equiv 2$ on $[3, \infty), 0 \leq \eta^{\prime} \leq 1$ and $\eta^{\prime \prime} \leq 0$ on $\mathbb{R}$. Set $\eta_{\alpha}(t)=\alpha \eta\left(\frac{t}{\alpha}\right)$ on $\mathbb{R}$ for any $\alpha>0$. Assume that $\Omega$ is a domain in $\Sigma$ with positive mean curvature boundary $\partial \Omega$. Then there is an area-minimizing hypersurface $S_{\lambda, \alpha}$ with the weight $e^{-\lambda \eta_{\alpha}\left(x_{n+1}\right)}$ in $\Omega \times \mathbb{R}$ with $\partial S_{\lambda, \alpha}=\partial \Omega \times\{0\}$ for $\lambda>0$. Note that $\eta_{\alpha}$ has a finite upper bound, so it is not hard to see that $S_{\lambda, \alpha}$ is compact. In particular, $S_{\lambda, \alpha}$ satisfies the following equation

$$
H+\lambda \eta_{\alpha}^{\prime}\left\langle E_{n+1}, \nu\right\rangle=0
$$

where $\nu$ is the unit normal vector of $S_{\lambda, \alpha}$. If $S_{\lambda, \alpha}$ is a graph over some open set with the graphic function $w$, the equation $(2.11)$ can be rewritten as

$$
\sum_{i, j=1}^{n}\left(\sigma^{i j}-\frac{w^{i} w^{j}}{1+|D w|^{2}}\right) w_{i, j}+\lambda \eta_{\alpha}^{\prime}(w)=0
$$

in a local coordinate.

Now let's show that $S_{\lambda, \alpha}$ is a graph over $\Omega$ by White's argument [18]. Let $S_{\lambda, \alpha}(t)=$ $\left\{X-t E_{n+1} \mid X \in S_{\lambda, \alpha}\right\}$ for any $t \in \mathbb{R}$. Let $t_{m}$ be the smallest nonnegative number such that $S_{\lambda, \alpha}\left(t_{m}\right)$ intersects $S_{\lambda, \alpha}$ in the interior. If $t_{m}=0$, then clearly $S_{\lambda, \alpha}$ is a graph. If $t_{m}>0$, the two hypersurfaces $S_{\lambda, \alpha}$ and $S_{\lambda, \alpha}\left(t_{m}\right)$ touch at an interior point $X=(x, t) \in \Omega \times \mathbb{R}$. Hence the tangent cones to $S_{\lambda, \alpha}$ and to $S_{\lambda, \alpha}\left(t_{m}\right)$ at $X$, respectively, both lie in halfspaces and are therefore a same hyperplane. So $X$ is a regular point of $S_{\lambda, \alpha}$ and $S_{\lambda, \alpha}\left(t_{m}\right)$, respectively. $S_{\lambda, \alpha}\left(t_{m}\right)$ satisfies the equation

$$
H+\lambda \eta_{\alpha}^{\prime}\left(\cdot+t_{m}\right)\left\langle E_{n+1}, \nu\right\rangle=0
$$

Namely, $S_{\lambda, \alpha}\left(t_{m}\right)$ can be written as a graph over some neighborhood of $x$ with a graphic function $\tilde{w}$ satisfying

$$
\sum_{i, j=1}^{n}\left(\sigma^{i j}-\frac{\tilde{w}^{i} \tilde{w}^{j}}{1+|D \tilde{w}|^{2}}\right) \tilde{w}_{i, j}+\lambda \eta_{\alpha}^{\prime}\left(\tilde{w}+t_{m}\right)=0 .
$$

Let $\varphi=w-\tilde{w}$, then $\varphi=0$ at $x$ and $\varphi \geq 0$ in some neighborhood of $x$ as $S_{\lambda, \alpha}\left(t_{m}\right)$ is below $S_{\lambda, \alpha}$. Moreover,

$$
\begin{aligned}
& \sum_{i, j}\left(\sigma^{i j}-\frac{\partial^{i} w \partial^{j} w}{1+|D w|^{2}}\right) \varphi_{i, j}=-\lambda \eta_{\alpha}^{\prime}(w)-\sum_{i, j}\left(\sigma^{i j}-\frac{\partial^{i} w \partial^{j} w}{1+|D w|^{2}}\right) \tilde{w}_{i, j} \\
&=-\lambda \eta_{\alpha}^{\prime}(w)+\lambda \eta_{\alpha}^{\prime}\left(\tilde{w}+t_{m}\right)+\sum_{i, j}\left(\frac{\partial^{i} w \partial^{j} w}{1+|D w|^{2}}-\frac{\partial^{i} \tilde{w} \partial^{j} \tilde{w}}{1+|D \tilde{w}|^{2}}\right) \tilde{w}_{i, j} \\
&=-\lambda \eta_{\alpha}^{\prime}(w)+\lambda \eta_{\alpha}^{\prime}\left(\tilde{w}+t_{m}\right)+\sum_{i, j}\left(\frac{\partial^{i} w \partial^{j} w-\partial^{i} \tilde{w} \partial^{j} \tilde{w}}{1+|D \tilde{w}|^{2}}+\frac{\partial^{i} w \partial^{j} w}{1+|D w|^{2}}-\frac{\partial^{i} w \partial^{j} w}{1+|D \tilde{w}|^{2}}\right) \tilde{w}_{i, j} \\
&=-\lambda \eta_{\alpha}^{\prime}(w)+\lambda \eta_{\alpha}^{\prime}\left(\tilde{w}+t_{m}\right)+\sum_{i, j} \frac{\partial^{i} \varphi \partial^{j} w+\partial^{i} \tilde{w} \partial^{j} \varphi}{1+|D \tilde{w}|^{2}} \tilde{w}_{i, j} \\
& \quad-\frac{\partial^{i} w \partial^{j} w \tilde{w}_{i, j}}{\left(1+|D w|^{2}\right)\left(1+|D \tilde{w}|^{2}\right)}\langle D(w+\tilde{w}), D \varphi\rangle .
\end{aligned}
$$

Combining $\eta_{\alpha}^{\prime \prime} \leq 0$ and $t_{m}>0$, we conclude that $S_{\lambda, \alpha}$ and $S_{\lambda, \alpha}\left(t_{m}\right)$ lie in parallel vertical planes by the strong maximum principle at $x$. However, it is impossible. So $S_{\lambda, \alpha}$ is a smooth graph over $\Omega$, which minimizes the weighted area with the weight $e^{-\lambda \eta_{\alpha}\left(x_{n+1}\right)}$ and with boundary $\partial \Omega \times\{0\}$ in $\Omega \times \mathbb{R}$. Moreover, if $u_{\lambda, \alpha}$ is the graphic function of $S_{\lambda, \alpha}$, then $u_{\lambda, \alpha} \geq 0$ by maximum principle. 
Set $d(x)=d(x, \partial \Omega)$ for all $x \in \Omega$, and $\Omega_{t}=\{x \in \Omega \mid d(x)>t\}$ for $t \geq 0$. There is a constant $0<\epsilon<1$ such that $\partial \Omega_{t}$ is smooth with mean curvature $\mathscr{H}(x, t) \geq \epsilon$ for any $x \in \partial \Omega_{t}$ and $0 \leq t<\epsilon$, and $d$ is smooth on $\Omega \backslash \Omega_{\epsilon}$.

Lemma 2.1. Let $u_{\lambda, \alpha}$ be a smooth solution to (2.12) on $\bar{\Omega}$ with $u_{\lambda, \alpha}=0$ on $\partial \Omega$ for any $\lambda, \alpha>0$, then we have the following boundary gradient estimate:

$$
\left|D u_{\lambda, \alpha}\right| \leq \epsilon^{-1}(\lambda+1) \quad \text { on } \partial \Omega \text {. }
$$

Proof. Let $\left\{e_{i}\right\}_{i=1}^{n}$ be an orthonormal vector field tangent to $\partial \Omega_{t}$ at a considered point $x \in \partial \Omega_{t}$, and denote $e_{n}$ be the unit normal vector field to $\partial \Omega_{t}$ so that $e_{n}$ points into $\Omega_{t}$. Since $d$ is a constant on $\partial \Omega_{t}$, then at $x$ we get

$$
\sum_{i=1}^{n-1}\left(D_{e_{i}} D_{e_{i}}-\left(D_{e_{i}} e_{i}\right)^{T}\right) d=0
$$

and $\left(D_{e_{n}} D_{e_{n}}-D_{e_{n}} e_{n}\right) d=0$, where $(\cdots)^{T}$ denotes the projection into the tangent bundle of $\partial \Omega_{t}$. Hence at $x$ one has

$$
\begin{aligned}
\Delta_{\Sigma} d & =\sum_{i=1}^{n}\left(D_{e_{i}} D_{e_{i}}-D_{e_{i}} e_{i}\right) d=-\sum_{i=1}^{n-1}\left\langle D_{e_{i}} e_{i}, e_{n}\right\rangle D_{e_{n}} d+\left(D_{e_{n}} D_{e_{n}}-D_{e_{n}} e_{n}\right) d \\
& =-\sum_{i=1}^{n-1}\left\langle D_{e_{i}} e_{i}, e_{n}\right\rangle=-\mathscr{H}(x, t) .
\end{aligned}
$$

Set $\Phi=\phi(d)=-(\lambda+1) \log \left(1-\epsilon^{-1} d\right)$. Then $\phi^{\prime}=(\lambda+1)(\epsilon-d)^{-1}$ and $\phi^{\prime \prime}=$ $(\lambda+1)(\epsilon-d)^{-2}$. Together with $(2.3),(2.16)$ and $\mathscr{H} \geq \epsilon$, on $\Omega \backslash \Omega_{\epsilon}$ we conclude that

$$
\begin{aligned}
& \operatorname{div}_{\Sigma}\left(\frac{D \Phi}{\sqrt{1+|D \Phi|^{2}}}\right)=\operatorname{div}_{\Sigma}\left(\frac{\phi^{\prime} D d}{\sqrt{1+\left|\phi^{\prime}\right|^{2}}}\right)=\frac{\phi^{\prime}}{\sqrt{1+\left|\phi^{\prime}\right|^{2}}} \Delta_{\Sigma} d+\frac{\phi^{\prime \prime}}{\left(1+\left|\phi^{\prime}\right|^{2}\right)^{\frac{3}{2}}} \\
= & \frac{-\mathscr{H}}{\sqrt{1+(\lambda+1)^{-2}(\epsilon-d)^{2}}}+\frac{(\lambda+1)^{-2}(\epsilon-d)}{\left(1+(\lambda+1)^{-2}(\epsilon-d)^{2}\right)^{\frac{3}{2}}} \\
\leq & \frac{-\epsilon}{\sqrt{1+(\lambda+1)^{-2}(\epsilon-d)^{2}}}+\frac{\epsilon(\lambda+1)^{-2}}{\left(1+(\lambda+1)^{-2}(\epsilon-d)^{2}\right)^{\frac{3}{2}}} \leq \frac{-\lambda(\lambda+1)^{-1} \epsilon}{\sqrt{1+(\lambda+1)^{-2}(\epsilon-d)^{2}}} \\
\leq & \frac{-\lambda(\lambda+1)^{-1}(\epsilon-d)}{\sqrt{1+(\lambda+1)^{-2}(\epsilon-d)^{2}}}=\frac{-\lambda}{\sqrt{1+|D \Phi|^{2}}} .
\end{aligned}
$$

Assume that $u_{\lambda, \alpha}$ is a smooth solution to $(2.12)$ on $\bar{\Omega}$ with $u_{\lambda, \alpha}=0$ on $\partial \Omega$ for any $\lambda, \alpha>0$. Analog to (2.14), $0 \leq u_{\lambda, \alpha} \leq \Phi$ on $\Omega \backslash \Omega_{\epsilon}$ by comparison principle. Then

$$
\left|D u_{\lambda, \alpha}\right| \leq|D \Phi|=\epsilon^{-1}(\lambda+1) \quad \text { on } \partial \Omega \text {. }
$$

For $\alpha>0$,

$$
\frac{\partial}{\partial \alpha} \eta_{\alpha}(t)=\eta\left(\frac{t}{\alpha}\right)-\frac{t}{\alpha} \eta^{\prime}\left(\frac{t}{\alpha}\right)=-\int_{0}^{\frac{t}{\alpha}} \tau \eta^{\prime \prime}(\tau) d \tau \geq 0
$$

Similar to (2.14), we conclude that $S_{\lambda, \beta}$ is above $S_{\lambda, \alpha}$ for any $\beta>\alpha$. Letting $\alpha \rightarrow \infty$, the graph $S_{\lambda, \alpha}$ converges to a generalized graph $S_{\lambda}$ over $\Omega$. Namely, let $u_{\lambda}=\lim _{\alpha \rightarrow \infty} u_{\lambda, \alpha} \geq 0$, then $S_{\lambda}=\partial\left\{(x, t) \mid u_{\lambda}(x)<t\right\}$ and $u_{\lambda}$ is the graphic function of $S_{\lambda}$. Note that $\left\{u_{\alpha}=\infty\right\}$ may be not empty, i.e., $S_{\lambda}$ may be not compact. Moreover, $S_{\lambda}$ is smooth and satisfies the 
equation (2.2) on $\left\{u_{\alpha}<\infty\right\}$. Obviously, $S_{\lambda}$ is an area-minimizing hypersurface with the weight $e^{-\lambda x_{n+1}}$ in $\Omega \times \mathbb{R}$ with boundary $\partial \Omega \times\{0\}$.

\section{Proof of the Main theOREM}

Let $\Omega$ be a bounded domain in an $n$-dimensional smooth manifold $\Sigma$ with smooth mean convex boundary. Assume that $\partial \Omega$ is not a minimal hypersurface in $\Sigma$. From [15], there is a mean curvature (level set) flow $\mathcal{M}: t \in[0, \infty) \mapsto M_{t}$ with $M_{0}=\partial \Omega$. By maximum principle, there is a sufficiently small constant $\epsilon_{0}>0$ such that $M_{t}$ has positive mean curvature everywhere for all $0<t \leq \epsilon_{0}$. Without loss of generality, we assume that $\partial \Omega$ has positive mean curvature everywhere.

Denote $F_{t}(\Omega)$ be a domain in $\Omega$ with $\partial F_{t}(\Omega)=M_{t}$ for $t \in[0, \infty)$. By [15], $F_{t}(\Omega)$ is mean convex for each $t \in[0, \infty)$, and $M_{t} \cap M_{t+\tau}=\emptyset, F_{t+\tau}(\Omega) \subset \operatorname{interior}\left(F_{t}(\Omega)\right)$ for all $0 \leq t<t+\tau<\infty$. Let $v: \bigcup_{t \geq 0} M_{t} \rightarrow \mathbb{R}$ be the function such that $v(x)=t$ for each $x \in M_{t}$. Then $v$ satisfies

$$
\operatorname{div}_{\Sigma}\left(\frac{D v}{|D v|}\right)+\frac{1}{|D v|}=0
$$

in the viscosity sense. Set $\Omega_{\infty}=\bigcap_{t>0} F_{t}(\Omega)$ and $M_{\infty}=\partial \Omega_{\infty}$. By [15], $M_{\infty}$ (maybe empty) has finitely many connected components, and the boundary of each component is a stable minimal variety whose singular set has Hausdorff dimension $\leq n-8$. Let the parabolic Hausdorff dimension of a set $E \subset \Sigma \times \mathbb{R}$ be the Hausdorff dimension of $E$ with respect to parabolic distance

$$
\operatorname{dist}_{P}((x, t),(y, \tau))=\max \left\{d(x, y),|t-\tau|^{1 / 2}\right\},
$$

where $d(\cdot, \cdot)$ is the distance function on $\Sigma$. Let $\widetilde{\mathcal{S}}$ be the spacetime singular set of $\mathcal{M}$ defined in [15]. Then the parabolic Hausdorff dimension of $\widetilde{\mathcal{S}}$ is at most $n-2$ by [15]. Denote $\mathcal{S}=\{x \in \Omega \mid$ there exists a $t>0$ such that $(x, t) \in \widetilde{\mathcal{S}}\}$.

Now we assume that $M_{\infty}$ is smooth. Then the mean curvature flow $M_{t}$ converges smoothly $M_{\infty}$ as $t \rightarrow \infty$. So there is an open set $K$ with $\bar{K} \subset \Omega \backslash \Omega_{\infty}$ such that $\overline{\mathcal{S}} \subset K$ and $v$ is smooth on $\bar{K} \backslash \mathcal{S}$.

From the previous argument, there is a translating soliton $S_{\lambda}$ whose graphic function $u_{\lambda}$ is a generalized function on $\Omega$ satisfying (2.3) on $\left\{u_{\lambda}<\infty\right\}$ and $u_{\lambda}=0$ on $\partial \Omega$ for any $\lambda>0$. Then

$$
t \in \mathbb{R} \rightarrow\left(S_{\lambda}\right)_{t} \triangleq \operatorname{graph}\left(u_{\lambda}-\lambda t\right)
$$

is a family of smooth hypersurfaces in $\Omega \times \mathbb{R}$ moving by mean curvature. Analog to the proof of Theorem 3 in [18], set $U_{\lambda}: \Omega \times \mathbb{R} \rightarrow \mathbb{R}$ by

$$
U_{\lambda}(x, y)=\lambda^{-1}\left(u_{\lambda}(x)-y\right)
$$

and $U: \Omega \times \mathbb{R} \rightarrow \mathbb{R}$ by

$$
U(x, y)=v(x) .
$$

As $\lambda \rightarrow \infty$, the mean curvature flows $t \in[0, \infty) \rightarrow\left(S_{\lambda}\right)_{t}$ converge as brakke flows to the flow $t \rightarrow M_{t} \times \mathbb{R}$ by elliptic regularization [12] and uniqueness of viscosity solution $v$. Since $U_{\lambda}^{-1}(t) \cap\{y=\tau\}=\left(S_{\lambda}\right)_{t} \cap\{y=\tau\}$ and $v^{-1}(t) \cap\{y=\tau\}=M_{t}$ for all $t, \tau \geq 0$, then $U_{\lambda}$ converges as $\lambda \rightarrow \infty$ uniformly to $U$ on $\bar{K}$. Namely, $\lambda^{-1} u_{\lambda}$ converges uniformly to $v$

on $\bar{K}$. By the local regularity theorem in [17] (or by Brakke's regularity theorem in [2]), $\lambda^{-1} u_{\lambda}$ converges as $\lambda \rightarrow \infty$ to $v$ smoothly on $\bar{K} \backslash \mathcal{S}$. 
Let $H_{\lambda}$ be the mean curvature of $S_{\lambda}$. By

$$
H_{\lambda}=-\frac{\lambda}{\sqrt{1+\left|D u_{\lambda}\right|^{2}}}=-\frac{1}{\sqrt{\lambda^{-2}+\lambda^{-2}\left|D u_{\lambda}\right|^{2}}},
$$

and $\lambda^{-1} u_{\lambda}$ converges uniformly to $v$ on $\bar{K}$, there is a small constant $0<\delta<1$ independent of $\lambda \geq 1$ such that

$$
H_{\lambda} \leq-\delta \quad \text { on } \bar{K} \text { for every } \lambda \geq 1 .
$$

Denote $\left|A_{\lambda}\right|^{2}$ be the square norm of the second fundamental form of $S_{\lambda}$. Choose a local orthonormal frame field $\left\{e_{i}\right\}_{i=1}^{n}$ in $S_{\lambda}$, which is a normal basis at the considered point. Combining (2.9) and (2.10), for any constant $\gamma$ we obtain

$$
\begin{aligned}
\Delta\left(h_{i j}+\gamma H_{\lambda}\right)= & \left\langle\lambda E_{n+1}, \nabla\left(h_{i j}+\gamma H_{\lambda}\right)\right\rangle-\left(\left|A_{\lambda}\right|^{2}+\overline{\operatorname{Ric}}(\nu, \nu)\right)\left(h_{i j}+\gamma H_{\lambda}\right) \\
& +\bar{R}_{k i k p} h_{j p}+\bar{R}_{k j k p} h_{i p}+2 \bar{R}_{k i j p} h_{k p}+\bar{\nabla}_{k} \bar{R}_{\nu j i k}+\bar{\nabla}_{i} \bar{R}_{\nu k j k}
\end{aligned}
$$

on $K$. Obviously, $\left|A_{\lambda}\right|^{2} \geq \frac{1}{n}\left|H_{\lambda}\right|^{2} \geq \frac{1}{n} \delta^{2}$ on $\bar{K}$ by (3.2), then there is a positive constant $C_{0}$ depending only on $n, \delta,|R|$ and $|D R|$ on $\Omega$ such that

$$
\Delta\left(h_{i j}+\gamma H_{\lambda}\right) \geq\left\langle\lambda E_{n+1}, \nabla\left(h_{i j}+\gamma H_{\lambda}\right)\right\rangle-\left(\left|A_{\lambda}\right|^{2}+\overline{R i c}(\nu, \nu)\right)\left(h_{i j}+\gamma H_{\lambda}\right)-C_{0}\left|A_{\lambda}\right|
$$

on $K$. Here $|R|^{2}=\sum_{i, j, k, l}\left|R_{i j k l}\right|^{2}$ and $|D R|^{2}=\sum_{i, j, k, l, m}\left|D R_{i j k l, m}\right|^{2}$.

Lemma 3.1. There is a positive constant $\gamma_{\lambda}^{*} \geq 1$ depending only on $n, \delta,|R|,|D R|$ on $\Omega$ and $\inf _{\partial K}\left(\left|A_{\lambda}\right| H_{\lambda}^{-1}\right)$ such that

$$
-\frac{1}{\gamma_{\lambda}^{*}} H_{\lambda} \leq\left|A_{\lambda}\right| \leq-\gamma_{\lambda}^{*} H_{\lambda} \quad \text { on } \bar{K} .
$$

Proof. Let $\kappa_{1} \geq \kappa_{2} \cdots \geq \kappa_{n}$ be the principal curvatures of $S_{\lambda}$. Note that $\kappa_{1}=\sup _{|\xi|=1} A_{\lambda}(\xi, \xi)$, then $\kappa_{1}$ is a continuous function on $S_{\lambda}$. Further, for any $\gamma, \tilde{\gamma} \in \mathbb{R}$,

$$
\sup _{K}\left(\kappa_{1}+\gamma H_{\lambda}\right) \leq \sup _{K}\left(\kappa_{1}+\tilde{\gamma} H_{\lambda}\right)+\sup _{K}\left((\gamma-\tilde{\gamma}) H_{\lambda}\right),
$$

which implies that $\sup _{K}\left(\kappa_{1}+\gamma H_{\lambda}\right)$ is also a continuous function on $\gamma \in \mathbb{R}$. There is a constant $\gamma_{0}$ depending on $\inf _{\partial K}\left(\left|A_{\lambda}\right| H_{\lambda}^{-1}\right)$ such that

$$
\sup _{\partial K}\left(\kappa_{1}+\gamma_{0} H_{\lambda}\right)=0 .
$$

If $\gamma_{0}<0$, we reset $\gamma_{0}=0$. Then we choose a constant $\gamma_{1}$ such that

$$
\sup _{K}\left(\kappa_{1}+\gamma_{1} H_{\lambda}\right)=-1
$$

We assume $\gamma_{1}>\gamma_{0}+\frac{1}{\delta}$, or else we complete the proof. By $H_{\lambda} \leq-\delta$, on $\partial K$ we have

$$
\kappa_{1}+\gamma_{1} H_{\lambda}=\kappa_{1}+\gamma_{0} H_{\lambda}+\left(\gamma_{1}-\gamma_{0}\right) H_{\lambda} \leq\left(\gamma_{1}-\gamma_{0}\right) H_{\lambda} \leq-\left(\gamma_{1}-\gamma_{0}\right) \delta<-1 .
$$

Hence $\kappa_{1}+\gamma_{1} H$ attains its maximum at some point $x_{0}$ in the interior of $K$. Choose a local orthonormal frame $\left\{e_{i}\right\}$ near $x_{0}$ in $\Sigma$ which is normal at $x_{0}$, and denote $h_{i j}=h\left(e_{i}, e_{j}\right)$ as mentioned before. Let $\xi=\left.\sum_{i} \xi_{i} e_{i}\right|_{p_{0}}$ be a unit eigenvector of the second fundamental form corresponding to the eigenvalue $\kappa_{1}\left(x_{0}\right)$ at the point $x_{0}$, namely, $h(\xi, \xi)=\kappa_{1}\left(x_{0}\right)$. Then the smooth function $\left.\hat{\kappa}_{1} \triangleq \sum_{i, j} h_{i j}\right|_{x} \xi_{i} \xi_{j}$ attains the maximum $\kappa_{1}\left(x_{0}\right)$ at $x_{0}$ in a neighborhood of $x_{0}$. From (3.4), we obtain

$$
\Delta\left(\hat{\kappa}_{1}+\gamma H_{\lambda}\right) \geq\left\langle\lambda E_{n+1}, \nabla\left(\hat{\kappa}_{1}+\gamma H_{\lambda}\right)\right\rangle-\left(\left|A_{\lambda}\right|^{2}+\overline{R i c}(\nu, \nu)\right)\left(\hat{\kappa}_{1}+\gamma H_{\lambda}\right)-C_{0}\left|A_{\lambda}\right| .
$$


By maximum principle for (3.7), at $x_{0}$ we have

$$
0 \geq-\left(\left|A_{\lambda}\right|^{2}+\overline{\operatorname{Ric}}(\nu, \nu)\right)\left(\hat{\kappa}_{1}+\gamma_{1} H_{\lambda}\right)-C_{0}\left|A_{\lambda}\right|=\left|A_{\lambda}\right|^{2}+\overline{\operatorname{Ric}}(\nu, \nu)-C_{0}\left|A_{\lambda}\right| .
$$

Let $c_{0}=\min \left\{0,\left.\inf _{|\xi|=1, x \in \Omega} \operatorname{Ric}\right|_{x}(\xi, \xi)\right\}$. Then (3.8) implies that at $x_{0}$

$$
\left|A_{\lambda}\right| \leq \frac{C_{0}}{2}+\sqrt{\frac{C_{0}^{2}}{4}-c_{0}} \leq C_{0}+\sqrt{-c_{0}}
$$

On the other hand, by (3.2) at $x_{0}$ one has

$$
-1=\kappa_{1}+\gamma_{1} H_{\lambda} \leq\left|A_{\lambda}\right|+\gamma_{1} H_{\lambda} \leq\left|A_{\lambda}\right|-\gamma_{1} \delta .
$$

Combining (3.9)(3.10) and the assumption $\gamma_{1}>\gamma_{0}+\frac{1}{\delta}$, we obtain

$$
\gamma_{1} \leq \gamma_{0}+\frac{1}{\delta}\left(C_{0}+\sqrt{-c_{0}}+1\right)
$$

According to the definition of $\gamma_{1}$ and $\kappa_{i}, \kappa_{1}+\gamma_{1} H_{\lambda} \leq-1<0$ on $\bar{K}$, which implies that

$$
\kappa_{n}=H_{\lambda}-\sum_{i=1}^{n-1} \kappa_{i} \geq H_{\lambda}-(n-1) \kappa_{1} \geq\left(1+(n-1) \gamma_{1}\right) H_{\lambda} .
$$

Hence we complete the proof.

Due to

$$
\begin{aligned}
& \left\langle\bar{\nabla}_{\partial_{x_{i}}+\partial_{i} u_{\lambda} E_{n+1}}\left(\partial_{x_{j}}+\partial_{j} u_{\lambda} E_{n+1}\right), \frac{-D u_{\lambda}+E_{n+1}}{\sqrt{1+\left|D u_{\lambda}\right|^{2}}}\right\rangle \\
= & \left\langle D_{\partial_{x_{i}}} \partial_{x_{j}}, \frac{-D u_{\lambda}+E_{n+1}}{\sqrt{1+\left|D u_{\lambda}\right|^{2}}}\right\rangle+\partial_{x_{i}} \partial_{x_{j}} u_{\lambda}\left\langle E_{n+1}, \frac{-D u_{\lambda}+E_{n+1}}{\sqrt{1+\left|D u_{\lambda}\right|^{2}}}\right\rangle \\
= & \left(\partial_{x_{i}} \partial_{x_{j}} u_{\lambda}-\left\langle D_{\partial_{x_{i}}} \partial_{x_{j}}, D u_{\lambda}\right\rangle\right) \frac{1}{\sqrt{1+\left|D u_{\lambda}\right|^{2}}}=\frac{\left(u_{\lambda}\right)_{i, j}}{\sqrt{1+\left|D u_{\lambda}\right|^{2}}},
\end{aligned}
$$

we have

$$
\left|A_{\lambda}\right|^{2}=\sum_{i, j, k, l}\left(\sigma^{i j}-\frac{\partial^{i} u_{\lambda} \partial^{j} u_{\lambda}}{1+\left|D u_{\lambda}\right|^{2}}\right) \frac{\left(u_{\lambda}\right)_{j, k}}{\sqrt{1+\left|D u_{\lambda}\right|^{2}}}\left(\sigma^{k l}-\frac{\partial^{k} u_{\lambda} \partial^{l} u_{\lambda}}{1+\left|D u_{\lambda}\right|^{2}}\right) \frac{\left(u_{\lambda}\right)_{l, i}}{\sqrt{1+\left|D u_{\lambda}\right|^{2}}} .
$$

Now let's show the main theorem.

Theorem 3.2. Let $\mathcal{M}: t \in[0, \infty) \mapsto M_{t}$ be a mean curvature flow starting from a mean convex, smooth hypersurface in an n-dimensional complete smooth manifold $\Sigma$. If $\lim _{t \rightarrow \infty}\left(\cup_{s>t} M_{s}\right)$ (maybe empty) is a smooth hypersurface, then all the singularities of $\mathcal{M}$ have convex type.

Proof. Let $v$ be a viscosity solution to (3.1) on $\Omega \backslash \Omega_{\infty}$, then $|D v|>0$ on $K \backslash \mathcal{S}$. From (3.14), $H_{\lambda}$ converges to $\operatorname{div}_{\Sigma}\left(\frac{D v}{|D v|}\right)$, and

$$
\begin{aligned}
\left|A_{\lambda}\right|^{2} & =\sum_{i, j, k, l}\left(\sigma^{i j}-\frac{\partial^{i} U_{\lambda} \partial^{j} U_{\lambda}}{\lambda^{-2}+\left|D U_{\lambda}\right|^{2}}\right)\left(\sigma^{k l}-\frac{\partial^{k} U_{\lambda} \partial^{l} U_{\lambda}}{\lambda^{-2}+\left|D U_{\lambda}\right|^{2}}\right) \frac{\left(U_{\lambda}\right)_{j, k}\left(U_{\lambda}\right)_{l, i}}{\lambda^{-2}+\left|D U_{\lambda}\right|^{2}} \\
\rightarrow\left|A_{\infty}\right|^{2} & \triangleq \sum_{i, j, k, l}\left(\sigma^{i j}-\frac{\partial^{i} v \partial^{j} v}{|D v|^{2}}\right) \frac{v_{j, k}}{|D v|}\left(\sigma^{k l}-\frac{\partial^{k} v \partial^{l} v}{|D v|^{2}}\right) \frac{v_{l, i}}{|D v|} \quad \text { as } \lambda \rightarrow \infty
\end{aligned}
$$


on $K \backslash \mathcal{S}$ smoothly. Here $-\operatorname{div}_{\Sigma}\left(\frac{D v}{|D v|}\right)$ and $\left|A_{\infty}\right|^{2}$ are the mean curvature and the square norm of the second fundamental form for the level set of $v$ in $K \backslash \mathcal{S}$, repsectively. Since $\partial K \cap \overline{\mathcal{S}}=\emptyset$, we conclude that $\inf _{\partial K}\left(\left|A_{\lambda}\right| H_{\lambda}^{-1}\right)$ is uniformly bounded for any $\lambda \geq 1$, and then $\gamma_{\lambda}^{*}$ in Lemma 3.1 is bounded by an absolute constant $\gamma^{*}$ independent of $\lambda \geq 1$. Namely, by Lemma 3.1 we have

$$
-\frac{1}{\gamma^{*}} H_{\lambda} \leq\left|A_{\lambda}\right| \leq-\gamma^{*} H_{\lambda} \quad \text { on } \bar{K} .
$$

Hence we obtain that

$$
-\frac{1}{\gamma^{*}} \operatorname{div}_{\Sigma}\left(\frac{D v}{|D v|}\right) \leq\left|A_{\infty}\right| \leq-\gamma^{*} \operatorname{div}_{\Sigma}\left(\frac{D v}{|D v|}\right) \quad \text { on } K \backslash \mathcal{S} .
$$

According to appendix B in [18], we complete the proof.

(i) Assume that $\Sigma$ has nonnegative Ricci curvature in Theorem 3.2. From (2.6), we have

$$
\Delta\left\langle E_{n+1}, \nu\right\rangle=-\left\langle E_{n+1}, \nabla H\right\rangle-\left(|A|^{2}+\overline{\operatorname{Ric}}(\nu, \nu)\right)\left\langle E_{n+1}, \nu\right\rangle .
$$

Let $S_{\lambda, \alpha}$ be a smooth graph with the graphic function $u_{\lambda, \alpha}$ satisfying (2.11). Combining (2.5), one has

$$
\Delta\left\langle E_{n+1}, \nu\right\rangle=\lambda \eta_{\alpha}^{\prime}\left\langle E_{n+1}, \nabla\left\langle E_{n+1}, \nu\right\rangle\right\rangle-\left(|A|^{2}+\overline{\operatorname{Ric}}(\nu, \nu)-\lambda \eta_{\alpha}^{\prime \prime}\left|\nabla u_{\lambda, \alpha}\right|^{2}\right)\left\langle E_{n+1}, \nu\right\rangle .
$$

Then by maximum principle for the above equation we have

$$
\sup _{\Omega} \sqrt{1+\left|D u_{\lambda, \alpha}\right|^{2}} \leq \sup _{\partial \Omega} \sqrt{1+\left|D u_{\lambda, \alpha}\right|^{2}}
$$

Combining the estimate (2.18), $\frac{1}{\lambda+1}\left|D u_{\lambda, \alpha}\right|$ is uniformly bounded on $\Omega$ independent of $\lambda, \alpha>0$ as well as $\frac{1}{\lambda+1} u_{\lambda, \alpha}$. Hence the graph $u_{\lambda}$ is a bounded graph with $u_{\lambda}=\lim _{\alpha \rightarrow \infty} u_{\lambda, \alpha}$. Since $\frac{1}{\lambda} u_{\lambda}$ converges to $v$ as $\lambda \rightarrow \infty$ on any compact set $Q$ in $\Omega \backslash \Omega_{\infty}$, we get that $v$ is bounded on $Q$ by a constant independent of $Q$. Hence the mean curvature flow $\mathcal{M}$ in Theorem 3.2 must vanish in finite time.

(ii) If $\Sigma$ is simply connected with nonpositive sectional curvature in Theorem 3.2, then we claim

$$
\sup _{t \in(0, \infty)}\left((1+t)^{-1} \sup _{x \in \Omega} u_{t}(x)\right)<\infty .
$$

Let's prove it by contradiction. If (3.19) fails, there are sequence $t_{i}>0$ and $\alpha_{i} \rightarrow \infty$ such that $\left(1+t_{i}\right)^{-1} \sup _{\Omega} u_{t_{i}, \alpha_{i}} \rightarrow \infty$ as $i \rightarrow \infty$, where $u_{t_{i}, \alpha_{i}}$ is a smooth solution to (2.12) with $\lambda, \alpha$ replaced by $t_{i}, \alpha_{i}$, respectively. We define $s_{i} \triangleq \sup _{\Omega} u_{t_{i}, \alpha_{i}}$ and $\widehat{u}_{t_{i}}=s_{i}^{-1} u_{t_{i}, \alpha_{i}}$, then

$$
\operatorname{div}_{\Sigma}\left(\frac{D \widehat{u}_{t_{i}}}{\sqrt{s_{i}^{-2}+\left|D \widehat{u}_{t_{i}}\right|^{2}}}\right)+\frac{t_{i} \eta_{\alpha_{i}}^{\prime}}{s_{i} \sqrt{s_{i}^{-2}+\left|D \widehat{u}_{t_{i}}\right|^{2}}}=0 .
$$

On the other hand, there is a point $x_{t_{i}} \in \Omega$ such that $\widehat{u}_{t_{i}}\left(x_{t_{i}}\right)=1$. Let $\rho_{x_{t_{i}}}(x)=d\left(x, x_{t_{i}}\right)$ for any $x \in \Omega$, then $\rho_{x_{t_{i}}}^{2}$ is smooth on $\Sigma$. By Hessian comparison theorem, we have

$$
\Delta_{\Sigma} \rho_{x_{t_{i}}}^{2} \geq 2 n
$$


Set $\Lambda=2 \operatorname{diam}(\Omega)>0$. Note $\left(1+t_{i}\right)^{-1} s_{i} \rightarrow \infty$ as $i \rightarrow \infty$. Hence for sufficiently large $i>0$

$$
\begin{aligned}
& \operatorname{div}_{\Sigma}\left(\frac{D\left(\frac{1}{2}-\Lambda^{-2} \rho_{x_{t_{i}}}^{2}\right)}{\sqrt{s_{i}^{-2}+\left|D\left(\frac{1}{2}-\Lambda^{-2} \rho_{x_{t_{i}}}^{2}\right)\right|^{2}}}\right)+\frac{t_{i}}{s_{i} \sqrt{s_{i}^{-2}+\left|D\left(\frac{1}{2}-\Lambda^{-2} \rho_{x_{t_{i}}}^{2}\right)\right|^{2}}} \\
\leq & \frac{-\Lambda^{-2} \Delta_{\Sigma} \rho_{x_{t_{i}}}^{2}}{\sqrt{s_{i}^{-2}+4 \Lambda^{-4} \rho_{x_{t_{i}}}^{2}}}+\frac{8 \Lambda^{-6} \rho_{x_{t_{i}}}^{2}}{\left(s_{i}^{-2}+4 \Lambda^{-4} \rho_{x_{t_{i}}}^{2}\right)^{\frac{3}{2}}}+\frac{t_{i}}{s_{i} \sqrt{s_{i}^{-2}+4 \Lambda^{-4} \rho_{x_{t_{i}}}^{2}}} \\
\leq & -\frac{2 n \Lambda^{-2}}{\sqrt{s_{i}^{-2}+4 \Lambda^{-4} \rho_{x_{t_{i}}}^{2}}}+\frac{t^{-6} \rho_{x_{t_{i}}}^{2}}{\left(s_{i}^{-2}+4 \Lambda^{-4} \rho_{x_{t_{i}}}^{2}\right)^{\frac{3}{2}}}+\frac{t_{i}}{s_{i} \sqrt{s_{i}^{-2}+4 \Lambda^{-4} \rho_{x_{t_{i}}}^{2}}} \\
\leq & \frac{-2(n-1) \Lambda^{-2} s_{i}+t_{i}}{s_{i} \sqrt{s_{i}^{-2}+4 \Lambda^{-4} \rho_{x_{t_{i}}}^{2}}}<0 .
\end{aligned}
$$

Let $\mathcal{E}$ be an open set defined by $\left\{x \in \Omega \mid \widehat{u}_{t_{i}}>\frac{1}{2}-\Lambda^{-2} \rho_{x_{t_{i}}}^{2}\right\}$. Since $\widehat{u}_{t_{i}}\left(x_{t_{i}}\right)=1$ and $\frac{1}{2}-\Lambda^{-2} \rho_{x_{t_{i}}}^{2}>0=\widehat{u}_{t_{i}}$ on $\partial \Omega$, then $\widehat{u}_{t_{i}}-\frac{1}{2}+\Lambda^{-2} \rho_{x_{t_{i}}}^{2}=0$ on $\partial \mathcal{E}$. Analog to (2.14), $\widehat{u}_{t_{i}}-\frac{1}{2}+\Lambda^{-2} \rho_{x_{t_{i}}}^{2}$ attains its maximum on $\mathcal{E}$ at the boundary $\partial \mathcal{E}$ by the maximum principle for (3.20) and (3.21). So we get a contradiction as $\widehat{u}_{t_{i}}-\frac{1}{2}+\Lambda^{-2} \rho_{x_{t_{i}}}^{2}=0$ on $\partial \mathcal{E}$, and the claim (3.19) holds.

So $(1+t)^{-1} \sup _{\Omega}\left|u_{t}\right|$ is uniformly bounded independent of $t>0$, which implies that $v$ is bounded and the mean curvature flow $\mathcal{M}$ in Theorem 3.2 must vanish in finite time.

Therefore, from Theorem 3.2 we can get the following corollary.

Corollary 3.3. Let $\mathcal{M}: t \in[0, \infty) \mapsto M_{t}$ be a mean curvature flow starting from a mean convex, smooth hypersurface in an $n$-dimensional complete smooth manifold $\Sigma$. If either $\Sigma$ has nonnegative Ricci curvature or $\Sigma$ is simply connected with nonpositive sectional curvature, then all the singularities of $\mathcal{M}$ have convex type.

\section{REFERENCES}

1. B. Andrews, Noncollapsing in mean-convex mean curvature flow, Geom. Topol. 16(3) (2012), 14131418.

2. K. Brakke, The motion of a surface by its mean curvature, Mathematical Notes, 20. Princeton University Press, Princeton, N.J., 1978.

3. T. H. Colding, T. Ilmanen and W. P. Minicozzi II, Rigidity of generic singularities of mean curvature flow, preprint, arXiv:1304.6356.

4. T. H. Colding and W. P. Minicozii II, Generic mean curvature flow I: generic singularities, Ann. of Math. 175(2) (2012), no. 2, 755-833.

5. Tobias H. Colding and William P. Minicozzi II, The singular set of mean curvature flow with generic singularities, arXiv:1405.5187, 2014.

6. D. Gilbarg and N. Trudinger, Elliptic Partial Differential Equations of Second Order, Springer-Verlag, Berlin-New York, (1983).

7. Robert Haslhofer and Or Hershkovits, Singularities of mean convex level set flow in general ambient manifolds, arXiv:1508.00840.

8. R. Haslhofer and B. Kleiner, Mean curvature flow of mean convex hypersurfaces, arXiv:1304.0926.

9. G. Huisken and C. Sinestrari, Mean curvature flow singularities for mean convex surfaces, Calc. Var. Partial Differential Equations 8 (1999), 1-14.

10. Gerhard Huisken and Carlo Sinestrari, Convexity estimates for mean curvature flow and singularities of mean convex surfaces, Acta Math. 183 (1999), no. 1, 45-70. 
11. Gerhard Huisken and Carlo Sinestrari, Mean curvature flow with surgeries of two-convex hypersurfaces, Invent. Math. 175 (2009), no. 1, 137-221.

12. T. Ilmanen, Elliptic regularization and partial regularity for motion by mean curvature, Mem. Amer. Math. Soc., 108(520), 1994.

13. P. Li, Harmonic Functions and Applications to Complete Manifolds, University of California, Irvine, Preprint, March 16, 2004.

14. W. M. Sheng and X. J. Wang, Singularity profile in the mean curvature flow, Methods Appl. Anal. 16(2) (2009), 139-155.

15. Brian White, The size of the singular set in mean curvature flow of mean-convex sets, J. Amer. Math. Soc. 13 (2000), no. 3, 665-695.

16. Brian White, The nature of singularities in mean curvature flow of mean-convex sets, J. Amer. Math. Soc. 16 (2003), no. 1, 123-138.

17. Brian White, A local regularity theorem for mean curvature flow, Ann. of Math. 161(2)(2005), no. 3, 1487-1519.

18. Brian White, Subsequent singularities in mean-convex mean curvature flow, to appear in Calc. Var. Partial Differential Equations (2013), arXiv:1103.1469v3.

19. Y. L. Xin, The translating solitons of mean curavture flow, arXiv:1410.5063v1.

20. X. P. Zhu, Lectures on mean curvature flows, AMS/IP Studies in Advanced Mathematics, 32, American Mathematical Society, Providence, RI; International Press, Somerville, MA, (2002).

Shanghai Center for Mathematical Sciences, Fudan University, Shanghai 200433, China

E-mail address: dingqi@fudan.edu.cn

E-mail address: dingqi09@fudan.edu.cn 